\title{
A Heavy Scalar at the LHC from Vector-Boson Fusion
}

\author{
Qiurong Mou and Sibo Zheng \\ Department of Physics, Chongqing University, Chongqing 401331, China \\ Correspondence should be addressed to Sibo Zheng; sibozheng.zju@gmail.com \\ Received 11 May 2018; Revised 3 July 2018; Accepted 26 July 2018; Published 2 August 2018 \\ Academic Editor: Enrico Lunghi
}

Copyright (C 2018 Qiurong Mou and Sibo Zheng. This is an open access article distributed under the Creative Commons Attribution License, which permits unrestricted use, distribution, and reproduction in any medium, provided the original work is properly cited. The publication of this article was funded by SCOAP ${ }^{3}$.

A hypothetical scalar mixed with the standard model Higgs appears in few contexts of new physics. This study addresses the question what mass range is in the reach of $14 \mathrm{TeV}$ LHC given different magnitudes of mixing angle $\alpha$, where event simulations are based on production from vector-boson fusion channel and decays into SM leptons through $W W$ or ZZ. It indicates that heavy scalar mass up to $539 \mathrm{GeV}$ and $937 \mathrm{GeV}$ can be excluded by integrated luminosity of $300 \mathrm{fb}^{-1}$ and $3000 \mathrm{fb}^{-1}$, respectively, for $\sin ^{2} \alpha$ larger than 0.04 .

\section{Introduction}

After the discovery of Higgs scalar $h$ at the LHC $[1,2]$, Standard Model (SM) as the effective field theory (EFT) of weak scale is established. While this EFT has not been violated at nowadays astrophysical and collider experiments, it must be incomplete in the light of a few indirectly experimental as well as theoretic hints. One of most robustly experimental hints arises from Plank and WIMP data [3], which suggests that there should be a new particle beyond SM serving as the thermal dark matter. On the other hand, one of theoretic challenges is the need of some novel mechanism to stabilize the divergence involving Higgs mass.

In a few new physics models attempted to complete the EFT of SM such as SM with doublets and supersymmetry, there usually exists a new scalar $H$ of the same spin, parity, and quantum numbers with SM Higgs but with heavier mass. Unless forbidden by some hidden symmetry, it generally mixes with the SM Higgs. If so, such scalar may leave signatures at dark matter facilities which are in the reach of $\mathrm{TeV}$ mass scale. See, e.g., [4-6] for very recent studies on this subject.

Alternatively, $H$ can mix with the SM Higgs and be examined at the LHC. Due to mixing effect $H$ couplings to SM particles are similar to those of SM Higgs but with an universal scaling factor related to mixing angle smaller than unity. As a result, the diboson decay channels $H \longrightarrow V_{i} V_{i}$ with $V_{i}$ referring to $W$ or $Z$ boson dominate others for $H$ mass above $200 \mathrm{GeV}$. In this case, $H$ is mainly generated at the LHC through gluon-gluon fusion (GGF) and vector-boson fusion (VBF) channels similar to the SM Higgs [7]. Early constraints [8-10] on the model parameters were obtained according to measurements on SM Higgs couplings, decay width, and direct detection at the LHC. Updated analyses based on the 8 $\mathrm{TeV}$ LHC data [11-17] can be found in [18-23].

In this paper, we will employ the techniques reported in [15] and study the prospect for the discovery of $H$ at the $14 \mathrm{TeV}$ LHC through processes of VBF production and subsequent diboson decays to SM leptons final states (The analysis here is more general than in the earlier version, which focused on the model interpretations of diboson excess reported in [24].). One reason is that although the GGF channel yields larger contribution to the production cross section than the VBF channel, the contribution to SM background cross section arising from GGF process is also larger than VBF process. Moreover, the ratio between GGF and VBF contribution to the production cross section declines from about $\sim 10$ to $\sim 2.5$ when $m_{h_{2}}$ increases from 200 $\mathrm{GeV}$ to $1 \mathrm{TeV}$.

The paper is organized as follows. In Section 2, we briefly discuss general parameterization of mixing effect in a model-independent way. The key point is that only two model parameters appear in the following study of direct detection. In Section 3, we address the production cross sections $\sigma(p p \longrightarrow H+X) \times \mathrm{Br}\left(H \longrightarrow V_{i} V_{i} \longrightarrow l \nu l v\right)$ from VBF channel at the LHC for $H$ mass above $200 \mathrm{GeV}$. Our 
main results are presented in Section 4 , where we show the luminosities required for the $2 \sigma$ exclusion and $5 \sigma$ discovery. Finally we conclude in Section 5.

\section{Model}

Without mixing effect such as in the case of scalar dark matter model, the mass squared matrix $\mathscr{M}^{2}$ for state vector $(H, h)$ reads as

$$
\mathscr{M}^{2}=\left(\begin{array}{cc}
m_{H}^{2} & 0 \\
0 & m_{h}^{2}
\end{array}\right)
$$

where $m_{H}$ and $m_{h}$ denote the mass of $H$ and $h$, respectively. There is little chance for direct detection on this kind of scalar at the LHC [25]. In contrast, in the case of mixing effect mass squared matrix $\mathscr{M}^{2}$ in (1) should be replaced with

$$
\mathscr{M}^{2}=\left(\begin{array}{cc}
m_{H}^{2} & \Delta m^{2} \\
\Delta m^{2} & m_{h}^{2}
\end{array}\right)
$$

where $\Delta m^{2}$ characterizes the mixing effect.

At present status only small mixing effect is allowed based on the LHC searches such as dijet, diphoton, and four-lepton signals. The mass eigenvalues can be approximated to be (Note that the analytic rather than approximations here will be utilized for the numerical calculation in the next section.)

$$
\begin{aligned}
& m_{h_{2}}^{2} \simeq m_{H}^{2}+\frac{\left(\Delta m^{2}\right)^{2}}{m_{H}^{2}-m_{h}^{2}}, \\
& m_{h_{1}}^{2} \simeq m_{h}^{2}-\frac{\left(\Delta m^{2}\right)^{2}}{m_{H}^{2}-m_{h}^{2}},
\end{aligned}
$$

together with their couplings to SM particles relative to SM Higgs

$$
\begin{aligned}
& \frac{g_{h_{1} X X}^{2}}{g_{h_{S M} X X}^{2}} \simeq \cos ^{2} \alpha, \\
& \frac{g_{h_{2} X X}^{2}}{g_{h_{S M} X X}^{2}} \simeq \sin ^{2} \alpha,
\end{aligned}
$$

Here, $X$ refers to the $\mathrm{SM}$ vector bosons and fermions, and the mixing angle $\alpha$ is given by

$$
\tan (2 \alpha) \simeq \frac{2 \Delta m^{2}}{m_{H}^{2}-m_{h}^{2}} .
$$

From (3) to (5) one finds that the productions and decays of these two scalars are totally determined by heavier mass $m_{h_{2}}$ and mixing angle $\sin \alpha$ after identifying $h_{1}$ as the SMlike Higgs. The magnitude of $\sin ^{2} \alpha$ has been up bounded to be less than $\sim 0.2$ at $95 \%$ CL in the light of precise measurement $[9,10]$ on the SM Higgs couplings at the 8 $\mathrm{TeV}$ LHC, and it will be improved to be of order $\sim 0.04$ at the future $14 \mathrm{TeV}$ LHC with designed integrated luminosity [26].

\section{Vector-Boson Fusion}

In this section, we address event simulation for the production cross section $\sigma\left(p p \longrightarrow h_{2}+X\right)$ from VBF channel and branching ratios $\operatorname{Br}\left(h_{2} \longrightarrow V_{i} V_{i} \longrightarrow l \nu l v\right)$ at the $14 \mathrm{TeV}$ LHC. In particular, we use package FeynRules [27] to generate model files prepared for MadGraph5 [28], which includes Pythia 6 [29] for parton showering and hadronization and the package Delphes 3 [30] for fast detector simulation.

3.1. Production Cross Section. We show in Figure 1 the strengths of cross sections for two different four-lepton final states, where the dependence on the mixing angle can be understood as follows. Firstly, according to (4), the VBF induced cross section $\sigma\left(p p \longrightarrow h_{2}\right)$ is proportional to $\sin ^{2} \alpha$. Secondly, with the definition on branching ratios $\operatorname{Br}\left(h_{2} \longrightarrow\right.$ $\left.V_{i} V_{i}\right)=\Gamma\left(h_{2} \longrightarrow V_{i} V_{j}\right) / \Gamma_{h_{2}}$, where $\Gamma_{h_{2}}=\Gamma\left(h_{2} \longrightarrow\right.$ $\mathrm{XX})+\Gamma\left(h_{2} \longrightarrow h_{1} h_{1}\right)$ ( $X$ is a SM fermion or vector boson), $\operatorname{Br}\left(h_{2} \longrightarrow V_{i} V_{i}\right)$ depends on the magnitude of $\Gamma\left(h_{2} \longrightarrow h_{1} h_{1}\right)$ relative to $\Gamma\left(h_{2} \longrightarrow \mathrm{XX}\right)$. Unlike $\Gamma\left(h_{2} \longrightarrow \mathrm{XX}\right)$ which is determined by the mixing effects in quadratic term of scalar potential, $\Gamma\left(h_{2} \longrightarrow h_{1} h_{1}\right)$ is directly related to the cubic term in the scalar potential, which is model-dependent. For example, in the minimal supersymmetric standard model, the ratio $\Gamma\left(h_{2} \longrightarrow h_{1} h_{1}\right) / \Gamma\left(h_{2} \longrightarrow \mathrm{XX}\right)$ is small for $m_{h_{2}}$ above $300 \mathrm{GeV}$ [31], which implies that $\operatorname{Br}\left(h_{2} \longrightarrow V_{i} V_{i}\right)$ mildly depends on the mixing angle. In contrast, $\Gamma\left(h_{2} \longrightarrow h_{1} h_{1}\right)$ can be important in models such as extended Higgs doublet models, where $\operatorname{Br}\left(h_{2} \longrightarrow V_{i} V_{i}\right)$ will be related to parameters such as mixing angle and quadratic and cubic terms in the scalar potential. For simplicity, we consider the case in which $\Gamma\left(h_{2} \longrightarrow h_{1} h_{1}\right)$ can be ignored.

The parameter space composed of mixing angle and heavy scalar mass is subject to both direct and indirect constraints. Current direct constraints include the $8 \mathrm{TeV}$ LHC bounds such as $\sigma_{\mathrm{GGF}+\mathrm{VBF}}\left(p p \longrightarrow h_{2}\right) \times \mathrm{Br}\left(h_{2} \longrightarrow \mathrm{gg}\right) \leq 200$ $\mathrm{fb}[32,33]$ and $\sigma_{\mathrm{GGF}+\mathrm{VBF}}\left(p p \longrightarrow h_{2}\right) \times \operatorname{Br}\left(h_{2} \longrightarrow \gamma \gamma\right) \leq 0.5 \mathrm{fb}$ [34] in the mass region below $200 \mathrm{GeV}$, as well as $\sigma_{\mathrm{VBF}}(p p \longrightarrow$ $\left.h_{2}\right) \times \mathrm{Br}\left(h_{2} \longrightarrow \mathrm{ZZ}\right)[16]$ and $\sigma_{\mathrm{VBF}}\left(p p \longrightarrow h_{2}\right) \times \mathrm{Br}\left(h_{2} \longrightarrow\right.$ WW) [17] in the mass region above $200 \mathrm{GeV}$. For illustration, we have shown in Figure 2 direct constraint on mixing angle in low mass region. On the other hand, indirect constraints include precision measures on the Yukawa couplings of SM Higgs $h_{1}$ to SM fermions and vector bosons. Global fits such as in [10] imply that $\sin ^{2} \alpha$ above 0.2 has been excluded. Other indirect constrains arising from measurements on precision electroweak observables may also be useful to constraint the mixing angle. In this sense, the constraint on mixing angle from indirect detection is much stronger than that from direct detection.

3.2. Events Selection. Let us now stress the event selections for the two different SM lepton final states from VBF channel. The primary SM backgrounds to the first process $h_{2} \longrightarrow$ $W W \longrightarrow l v l v$ include dilepton + jets and QCD multijets. For simplicity, we consider the main contributions arising from dilepton plus jets channels and adopt the cuts used by the CMS VBF analysis [15] for event selection: 

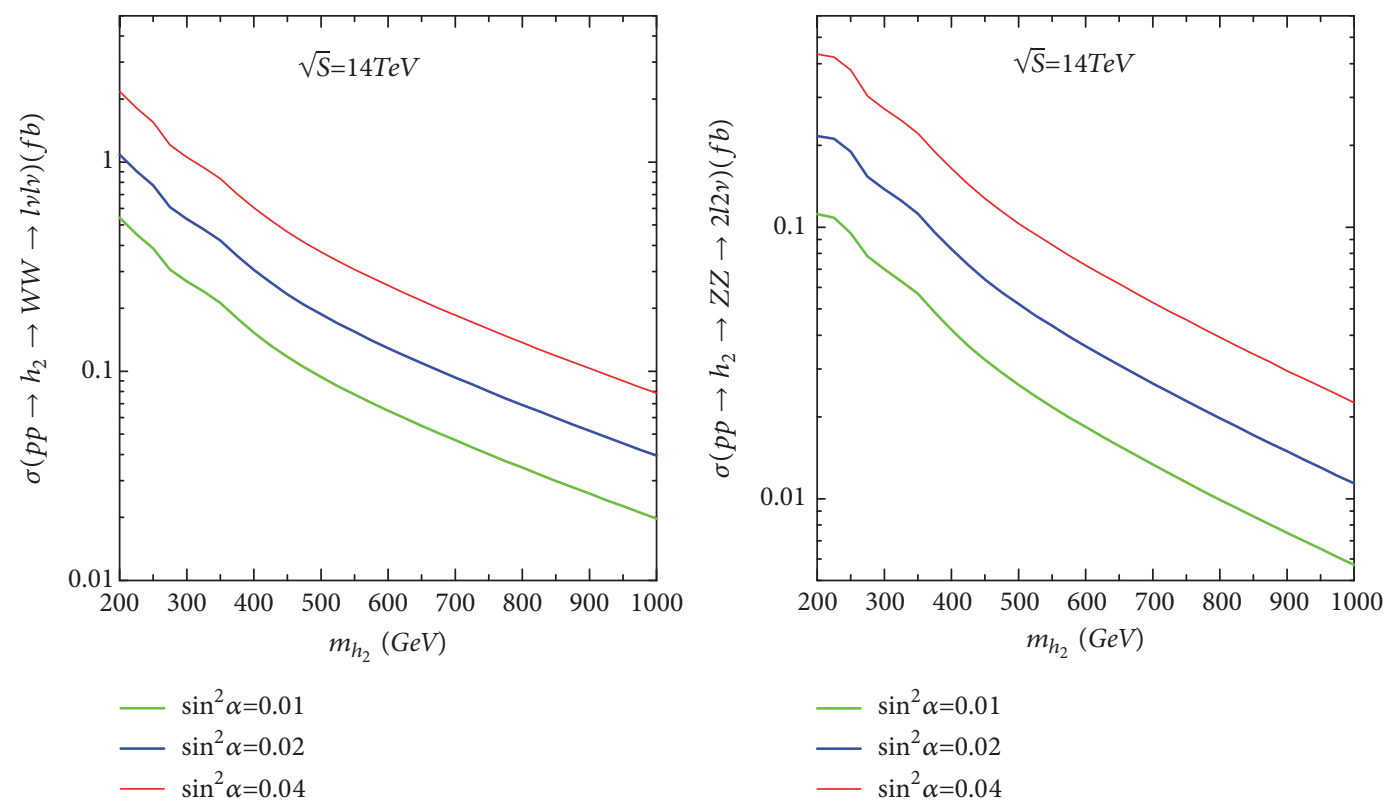

Figure 1: Production cross section $\sigma\left(p p \longrightarrow h_{2}+X\right) \times \operatorname{Br}\left(h_{2} \longrightarrow W W \longrightarrow l v l v\right)$ (left) and $\sigma\left(p p \longrightarrow h_{2}+X\right) \times \operatorname{Br}\left(h_{2} \longrightarrow Z Z \longrightarrow 2 l 2 v\right)$ (right) as function of $m_{h_{2}}$ for different strengths of mixing effect $\sin ^{2} \alpha=0.01$ (green), 0.02 (blue), and 0.04 (red), respectively.

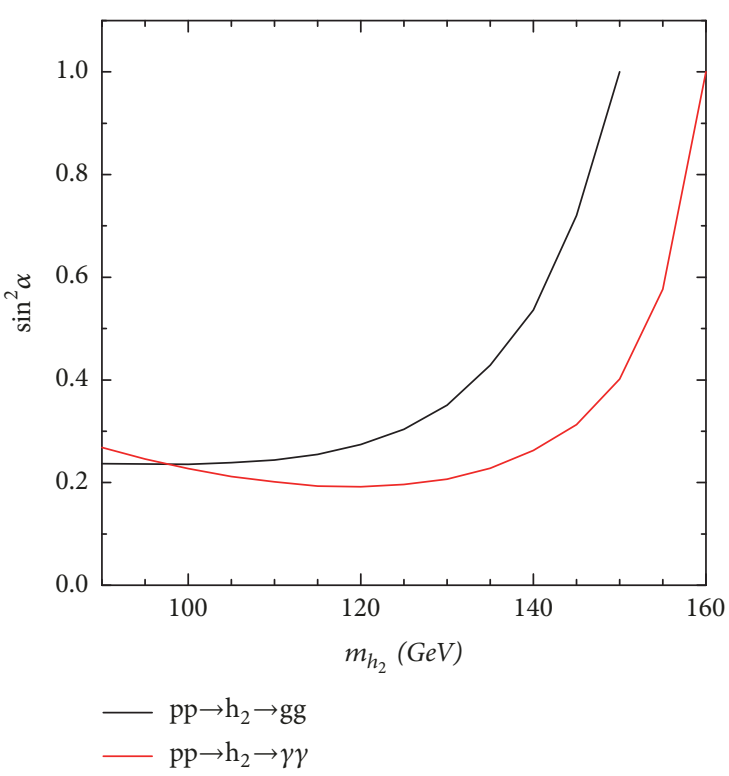

FIGURE 2: Direct constraint on mixing angle from decay modes $h_{2} \longrightarrow \gamma \gamma$ [34] and $h_{2} \longrightarrow$ gg $[32,33]$ at the $8 \mathrm{TeV}$ LHC, which is verified to be much weaker than indirect constraint from precision tests on SM Higgs.

$$
\begin{aligned}
\left|\eta_{\mu}\right| & <2.4, \\
\left|\Delta \eta_{j j}\right| & >3.5 \\
M_{l l} & >12 \mathrm{GeV}, \\
M_{j j} & >500 \mathrm{GeV}, \\
E_{\mathrm{T}, \mathrm{Pr}}^{\mathrm{miss}} & >20 \mathrm{GeV},
\end{aligned}
$$

where $p_{T}^{l_{1(2)}}$ and $p_{T}^{j_{1(2)}}$ are the transverse momentum of the first (second) leading lepton $l=\{e, \mu\}$ and jet, respectively; $\eta_{e(\mu)}$ is pseudorapidity of $e(\mu) ; \Delta \eta_{j j}$ and $M_{j j(l l)}$ is the rapidity difference and invariant mass of the two leading jets (leptons), respectively. Parameter $E_{\mathrm{T}, P r}^{\mathrm{m} i s s}$ is defined as

$$
E_{\mathrm{T}, \mathrm{Pr}}^{\mathrm{miss}}= \begin{cases}E_{T}^{\mathrm{miss}} \cdot \cos (\Delta \Phi), & \Delta \Phi\left(p_{T}, E_{T}^{\mathrm{miss}}\right)<\frac{\pi}{2} \\ \vec{E}_{T}^{\text {miss }}, & \Delta \Phi\left(p_{T}, E_{T}^{\mathrm{miss}}\right)>\frac{\pi}{2} .\end{cases}
$$

with $\Delta \Phi\left(p_{T}, E_{T}^{\text {miss }}\right)$ referring to the azimuthal angle between the dilepton transverse momentum and $\vec{E}_{T}^{\text {miss }}$. Any event with an additional jet with $p_{T}>30 \mathrm{GeV}$ is rejected. We refer the reader to [15] for more details.

For the second process $h_{2} \longrightarrow Z Z \longrightarrow 2 l 2 v$ we consider the main contributions arising from electron pair + jets + $E_{T}^{\text {miss }}$ and muon pair + jets $+E_{T}^{\text {miss }}$. Cuts [15] for event selection in this channel are given by

$$
\begin{array}{r}
p_{T}^{l 1(2)}>20 \mathrm{GeV}, \\
p_{T}^{l l}>55 \mathrm{GeV},
\end{array}
$$


TABLE 1: Effects on the ratio $S / B$ due to variations on the cuts in (6) (top) and (8) (bottom) for benchmark mass $m_{h 2}=600 \mathrm{GeV}$ at $14 \mathrm{TeV}$ LHC.

\begin{tabular}{lccc}
\hline$p_{T}^{l 1}>\{10,20,40\} \mathrm{GeV}$ & $p_{T}^{j 1}>\{400,500,600\} \mathrm{GeV}$ & $\Delta \eta_{j j}>\{3,3.5,4.0\}$ & $M_{j j}>\{400,500,600\} \mathrm{GeV}$ \\
$\{0.98,1,1.02\}$ & $\{1,1,0.99\}$ & $\{0.79,1,1.38\}$ & $\{0.82,1,1.36\}$ \\
\hline$p_{T}^{e}>\{10,20,40\} \mathrm{GeV}$ & $p_{T}^{j 1}>\{20,30,40\} \mathrm{GeV}$ & $\Delta \eta_{j j}>\{3.5,4,4.5\}$ & $M_{j j}>\{400,500,600\} \mathrm{GeV}$ \\
$\{1,1,0.99\}$ & $\{1,1,0.98\}$ & $\{1.51,1,0.7\}$ & $\{1.13,1,0.90\}$ \\
\hline
\end{tabular}
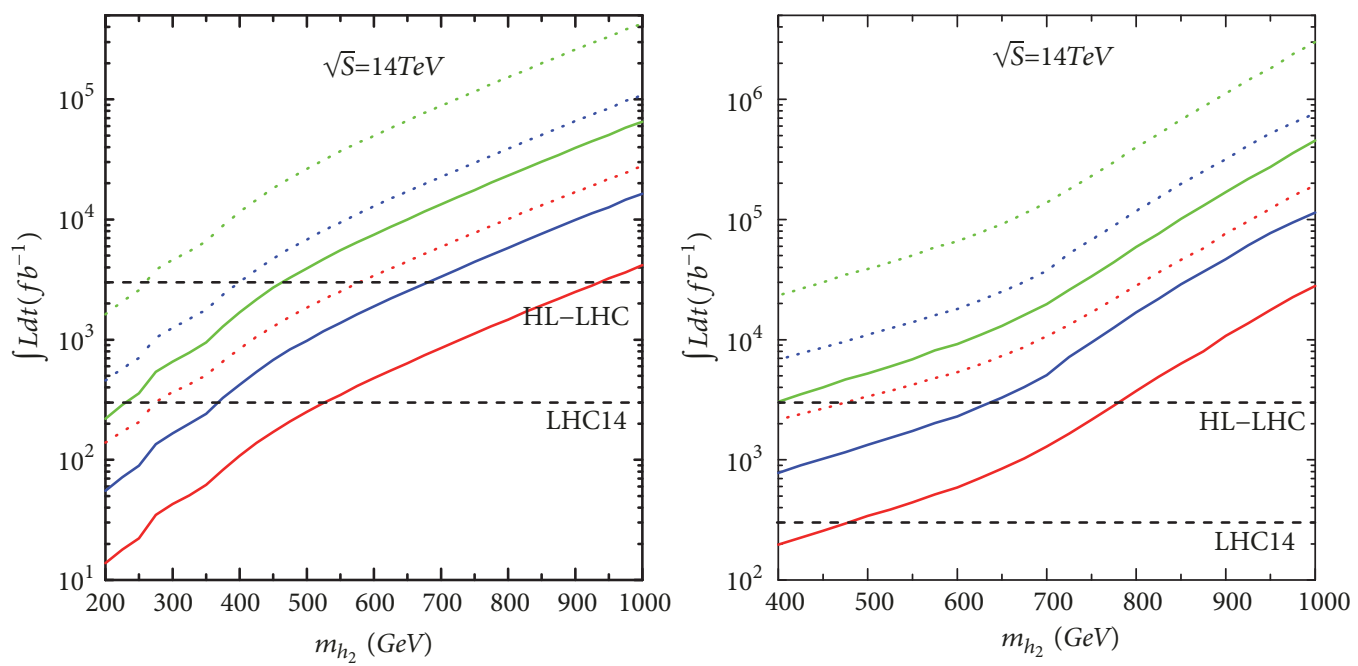

FIGURE 3: The integrated luminosity needed for the exclusion determined by $S / \sqrt{B}=1.96$ (solid) and $5 \sigma$ discovery determined by $S / \sqrt{S+B}=$ 5 (dotted) at the $14 \mathrm{TeV}$ LHC, respectively. Here, the left and right plot correspond to the left and right plot of Figure 1, respectively, where the

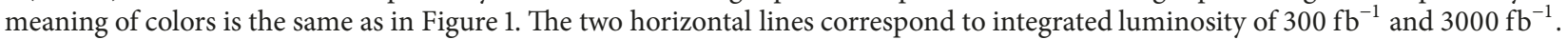

$$
\begin{aligned}
p_{T}^{j} & >30 \mathrm{GeV}, \\
\left|\eta_{j}\right| & <2.5, \\
\left|\Delta \eta_{j j}\right| & >4, \\
M_{j j} & >500 \mathrm{GeV}, \\
60 \mathrm{GeV} & <M_{l l}<120 \mathrm{GeV}, \\
E_{T}^{\mathrm{miss}} & \geq 70 \mathrm{GeV},
\end{aligned}
$$

where $p_{T}^{l l}$ denotes the $p_{T}$ of the dilepton system. Any event which includes the third lepton with $p_{T}>20 \mathrm{GeV}$ is rejected in order to suppress SM $W Z$ background. More details can be also found in [15].

Cuts in (6) and (8) will be applied to the $14 \mathrm{TeV}$ LHC simulations for conservation, the validity of which is guaranteed by the following facts. At first, there is little difference between the $8 \mathrm{TeV}$ LHC and $14 \mathrm{TeV}$ LHC except the collision energy, which means the cut on the pseudorapidity of the first two leading jets should remain unchanged. Second, the kinetic distribution of the signal events and the main SM backgrounds have similar changing trends when one modifies these cuts. Take the representative mass $m_{h_{2}}=$ $600 \mathrm{GeV}$ for example. The effects on the ratio of signal over background events $S / B$ are less than two times due to variations on the cuts in (6) and (8). See Table 1 for details.

\section{Results}

Following the definition $S / \sqrt{B}$ and $S / \sqrt{S+B}$ about significance for exclusion and discovery, respectively, we show the values of $L$ needed for exclusion and discovery at the $14 \mathrm{TeV}$ LHC in Figure 3. Systematic uncertainties are neglected in both the signal and the background simulations.

The left plot therein addresses the decay $h_{2} \longrightarrow W W \longrightarrow$ $l \nu l \nu$. In the left one, we observe that for $L=300 \mathrm{fb}^{-1} h_{2}$ mass up to $\{270,368,539\} \mathrm{GeV}$ can be excluded via final state $l \nu l \nu$ for $\sin ^{2} \alpha=\{0.01,0.02,0.04\}$, respectively; and the discovery limit approaches to $275 \mathrm{GeV}$ for $\sin ^{2} \alpha=0.04$. Furthermore, for HL-LHC with $L=3000 \mathrm{fb}^{-1}$ [26], $h_{2}$ mass up to $\{459,675,937\} \mathrm{GeV}$ can be excluded via this channel for $\sin ^{2} \alpha=\{0.01,0.02,0.04\}$, respectively; and the discovery limits reach $\{267,401,583\} \mathrm{GeV}$ for $\sin ^{2} \alpha=\{0.01,0.02,0.04\}$, respectively.

The right plot in Figure 3 addresses the decay $h_{2} \longrightarrow$ $Z Z \longrightarrow 2 l 2 v$. It shows that for $L=300 \mathrm{fb}^{-1} h_{2}$ mass up to $475 \mathrm{GeV}$ can be excluded through final state $2 l 2 v$ for $\sin ^{2} \alpha=0.04$. Moreover, for the HL-LHC $h_{2}$ mass up to $\{400,640,790\} \mathrm{GeV}$ can be excluded via the same channel for $\sin ^{2} \alpha=\{0.01,0.02,0.04\}$, respectively; and the discovery limit reaches $477 \mathrm{GeV}$ for $\sin ^{2} \alpha=0.04$. The exclusion limits via the $Z Z$ decay are relatively weaker in comparison with the $W W$ decay. 


\section{Conclusions}

Hypothetical scalar similar to the SM Higgs often appears in a complete model of quantum field theory. This work is devoted to study a heavy scalar mixed with SM Higgs at the $14 \mathrm{TeV}$ LHC through VBF channel. We have simulated events arising from diboson decays such as $h_{2} \longrightarrow W W \longrightarrow l v l v$ and $h_{2} \longrightarrow$ $Z Z \longrightarrow 2 l 2 v$, where both exclusion and discovery limits are revealed according to different magnitudes of mixing effect. Our study demonstrates that such type of heavy scalar with mass up to $539 \mathrm{GeV}$ and $937 \mathrm{GeV}$ can be excluded by the 14 $\mathrm{TeV}$ LHC with $L=300 \mathrm{fb}^{-1}$ and $3000 \mathrm{fb}^{-1}$, respectively, for $\sin ^{2} \alpha$ larger than 0.04 .

\section{Data Availability}

The data used to support the findings of this study are available from the corresponding author upon request.

\section{Conflicts of Interest}

The authors do not have a direct financial relation with any commercial identity mentioned in the paper that might lead to conflicts of interest for any of the authors.

\section{Acknowledgments}

This work is supported in part by the National Natural Science Foundation of China under Grant no. 11775039.

\section{References}

[1] G. Aad et al., "Observation of a new particle in the search for the Standard Model Higgs boson with the ATLAS detector at the LHC," Physics Letters B, vol. 716, no. 1, 2012.

[2] C. Mariotti, "Observation of a new boson at a mass of $125 \mathrm{GeV}$ with the CMS experiment at the Lhc," in Proceedings of the MG13 Meeting on General Relativity, pp. 352-372, Stockholm University, Sweden.

[3] Planck. Collaboration, "Planck 2013 results. XVI. Cosmological parameters," Astronomy \& Astrophysics, vol. 571, no. A16, 2013, arXiv:1303.5076.

[4] X. He and J. Tandean, "New LUX and PandaX-II results illuminating the simplest Higgs-portal dark matter models," Journal of High Energy Physics, vol. 2016, no. 12, 2016.

[5] H. Wu and S. Zheng, "Scalar dark matter: real vs complex," Journal of High Energy Physics, vol. 2017, no. 3, 2017.

[6] J. Casas, D. Cerdeño, J. Moreno, and J. Quilis, "Reopening the Higgs portal for single scalar dark matter," Journal of High Energy Physics, vol. 1705, no. 36, 2017.

[7] S. Dittmaier, "Handbook of LHC Higgs Cross Sections: 1. Inclusive Observables," LHC Higgs Cross Section Working Group, 2016.

[8] V. Barger, P. Langacker, M. McCaskey, M. J. Ramsey-Musolf, and G. Shaughnessy, "CERN LHC phenomenology of an extended standard model with a real scalar singlet," Physical Review D: Particles, Fields, Gravitation and Cosmology, vol. 77, no. 3, Article ID 035005, 2008.
[9] P. P. Giardino, K. Kannike, I. Masina, M. Raidal, and A. Strumia, "The universal Higgs fit," Journal of High Energy Physics, vol. 2014, no. 5, 2014.

[10] A. Falkowski, F. Riva, and A. Urbano, "Higgs at last," Journal of High Energy Physics, vol. 2013, no. 11, 2013.

[11] S. Chatrchyan et al., "Measurement of the properties of a Higgs boson in the four-lepton final state," Physical Review D, vol. 89, no. 9, Article ID 092007, 2014.

[12] V. Khachatryan et al., "Search for massive resonances decaying into pairs of boosted bosons in semi-leptonic final states at $s=8$ TeV," Journal of High Energy Physics, vol. 1408, 2014.

[13] V. Khachatryan et al., "Observation of the diphoton decay of the Higgs boson and measurement of its properties," European Physical Journal C, vol. 10, p. 3076, 2014, arXiv:1407.0558.

[14] G. Aad et al., "Search for Scalar Diphoton Resonances in the Mass Range 65-600 GeV with the ATLAS Detector in pp Collision Data at $s=8 \mathrm{TeV}$," Physical Review Letters, vol. 113, no. 17, 2014.

[15] V. Khachatryan et al., "Search for a Higgs boson in the mass range from 145 to $1000 \mathrm{GeV}$ decaying to a pair of W or Z bosons," Journal of High Energy Physics, vol. 1510, 2015.

[16] G Aad, "Search for an additional, heavy Higgs boson in the $\mathrm{H} \longrightarrow \mathrm{ZZ}$ decay channel at $\mathrm{s}=8 \mathrm{TeV}$ in pp collision data with the ATLAS detector," European Physical Journal C, vol. 76, no. 1, 2016.

[17] G. Aad et al., "Search for a high-mass Higgs boson decaying to a $\mathrm{W}$ boson pair in pp collisions at $\mathrm{s}=8 \mathrm{TeV}$ with the ATLAS detector," Journal of High Energy Physics, vol. 32, 2016.

[18] T. Robens and T. Stefaniak, "Status of the Higgs singlet extension of the standard model after LHC run 1," The European Physical Journal C, vol. 75, no. 3, article 104, 2015.

[19] A. Falkowski, C. Gross, and O. Lebedev, "A second Higgs from the Higgs portal," Journal of High Energy Physics, vol. 2015, no. $5,2015$.

[20] S. I. Godunov, A. N. Rozanov, M. I. Vysotsky, and E. V. Zhemchugov, "Extending the Higgs sector: an extra singlet," European Physical Journal C, vol. 76, no. 1, 2016.

[21] D. Buttazzo, F. Sala, and A. Tesi, "Singlet-like Higgs bosons at present and future colliders," Journal of High Energy Physics, vol. 2015, no. 11, 2015.

[22] K. Cheung, P. Ko, J. S. Lee, and P. Tseng, "Bounds on Higgsportal models from the LHC Higgs data," Journal of High Energy Physics, vol. 2015, no. 10, article 057, 2015.

[23] T. Robens and T. Stefaniak, "LHC benchmark scenarios for the real Higgs singlet extension of the standard model," The European Physical Journal C, vol. 76, article 268, 2016.

[24] G. Aad et al., "Search for high-mass diboson resonances with boson-tagged jets in proton-proton collisions at $\mathrm{s}=8 \mathrm{TeV}$ with the ATLAS detector," JHEP, vol. 1512, no. 055, 2015.

[25] H. Han, J. M. Yang, Y. Zhang, and S. Zheng, "Collider signatures of Higgs-portal scalar dark matter," Physics Letters B, vol. 756, pp. 109-112, 2016.

[26] S. Dawson et al., "Working Group Report: Higgs Boson,".

[27] A. Alloul, N. D. Christensen, C. Degrande, C. Duhr, and B. Fuks, "FeynRules 2.0-a complete toolbox for tree-level phenomenology," Computer Physics Communications, vol. 185, no. 8, pp. 2250-2300, 2014.

[28] J. Alwall, R. Frederix, and S. Frixione, "The automated computation of tree-level and next-to-leading order differential cross sections, and their matching to parton shower simulations," Journal of High Energy Physics, vol. 2014, no. 7, article 079, 2014. 
[29] T. Sjöstrand, S. Mrenna, and P. Skands, "PYTHIA 6.4 physics and manual," Journal of High Energy Physics, vol. 0605, no. 026, 2006.

[30] J. de Favereau, C. Delaere, P. Demin et al., "DELPHES 3: a modular framework for fast simulation of a generic collider experiment," Journal of High Energy Physics, vol. 2014, article 57, 2014.

[31] J. F. Gunion, H. E. Haber, G. L. Kane, and S. Dawson, “The Higgs hunter's guide," Frontiers of Physics, vol. 80, pp. 1-448, 2000.

[32] G. Aad et al., "Search for new phenomena in the dijet mass distribution using $\mathrm{p}$ - $\mathrm{p}$ collision data at $\mathrm{s}=8 \mathrm{TeV}$ with the ATLAS detector," Physical Review D, vol. 91, no. 5, 2015.

[33] V. Khachatryan et al., "Search for resonances and quantum black holes using dijet mass spectra in proton-proton collisions at $\mathrm{s}=8 \mathrm{TeV}$," Physical Review D, vol. 91, no. 5, 2015.

[34] G. Aad et al. [ATLAS Collaboration,arXiv:1504.05511 [hep-ex]]. 

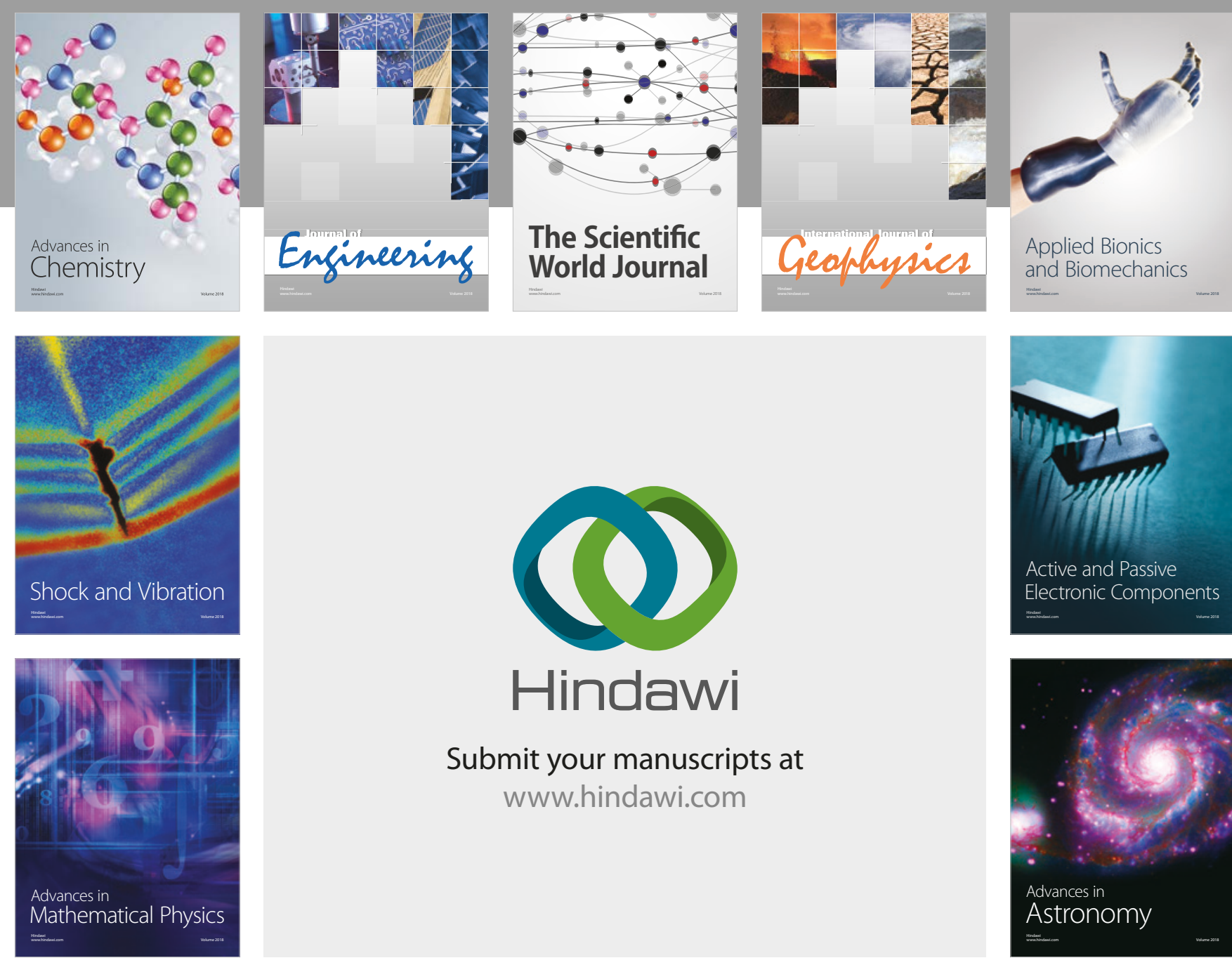

Submit your manuscripts at

www.hindawi.com

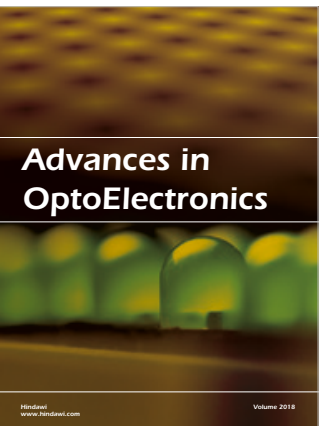

\section{Rotcting Machinery}
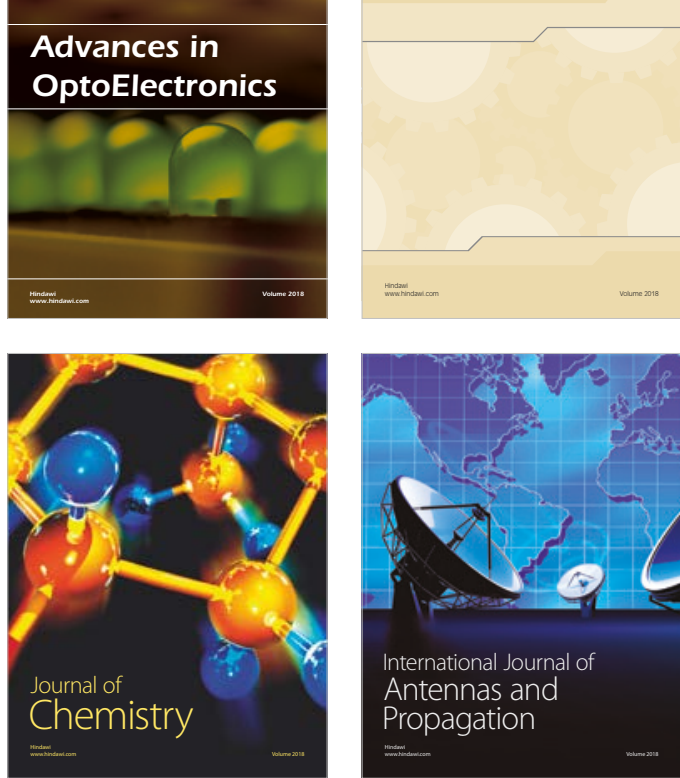

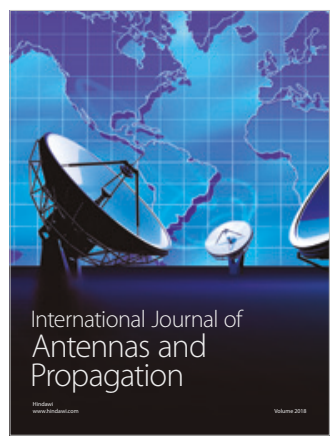

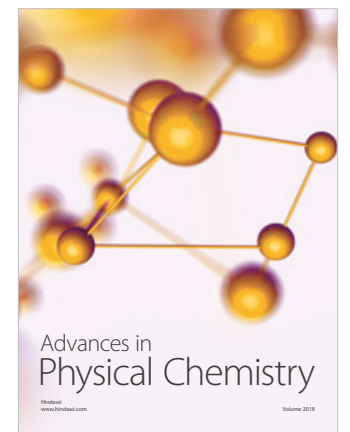

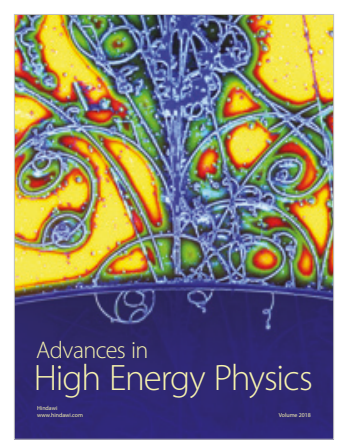

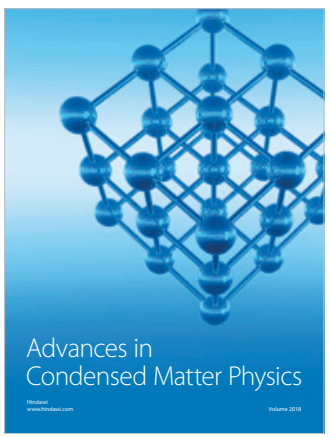

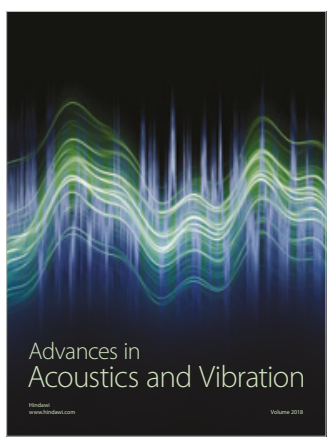

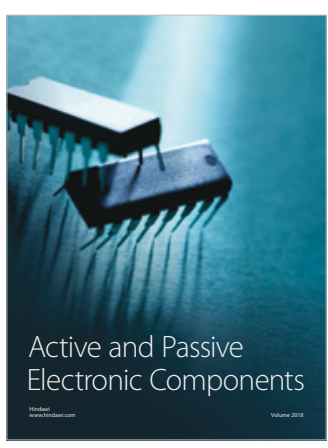
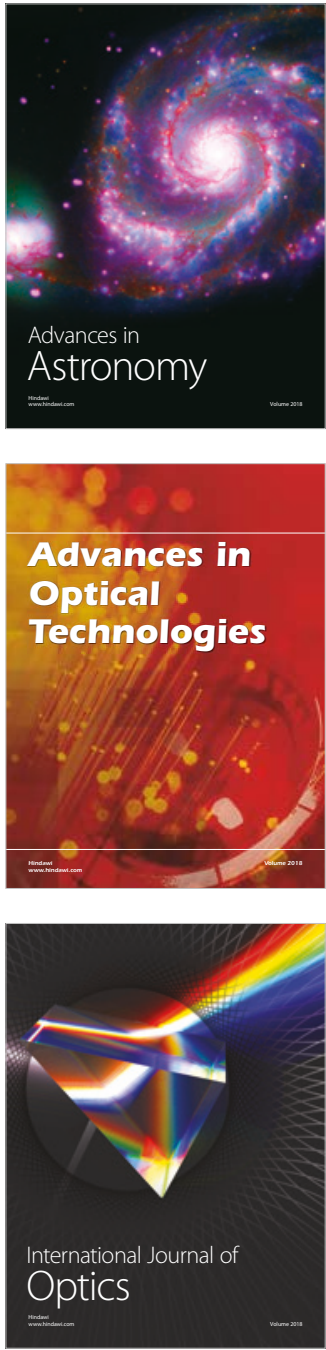